University of Nebraska - Lincoln

DigitalCommons@University of Nebraska - Lincoln

\title{
Variations in plantar pressure variables across elliptical trainers in older adults
}

Judith M. Burnfield

University of Nebraska-Lincoln, jburnfield@madonna.org

Guilherme Manna Cesar

University of Nebraska-Lincoln, g.cesar@unf.edu

Thad W. Buster

Madonna Rehabilitation Hospitals, tbuster@madonna.org

Follow this and additional works at: https://digitalcommons.unl.edu/mechengfacpub

Part of the Mechanics of Materials Commons, Nanoscience and Nanotechnology Commons, Other Engineering Science and Materials Commons, and the Other Mechanical Engineering Commons

Burnfield, Judith M.; Cesar, Guilherme Manna; and Buster, Thad W., "Variations in plantar pressure variables across elliptical trainers in older adults" (2020). Mechanical \& Materials Engineering Faculty Publications. 599.

https://digitalcommons.unl.edu/mechengfacpub/599

This Article is brought to you for free and open access by the Mechanical \& Materials Engineering, Department of at DigitalCommons@University of Nebraska - Lincoln. It has been accepted for inclusion in Mechanical \& Materials Engineering Faculty Publications by an authorized administrator of DigitalCommons@University of Nebraska Lincoln. 


\title{
Variations in plantar pressure variables across elliptical trainers in older adults
}

\author{
Judith M. Burnfield, Guilherme M. Cesar, \\ \& Thad W. Buster \\ Institute for Rehabilitation Science and Engineering, Madonna Rehabilitation Hospitals, \\ 5401 South Street, Lincoln, NE 68506, United States \\ Corresponding author - J. M. Burnfield, email jburnfield@madonna.org \\ E-mail addresses - gcesar@madonna.org (G.M. Cesar), tbuster@madonna.org (T.W. Buster)
}

\begin{abstract}
Background: Ellipticals are used to address walking and cardiorespiratory training goals of older adults, some of whom are at risk for foot injuries. Variations in joint kinematics and muscle demands when using different ellipticals could lead to plantar pressure differences. This study explored plantar pressure variables during gait and use of four ellipticals.

Methods: Plantar pressures were recorded while 10 adults [68.1 (4.5) years] walked and used the True, Octane, Life Fitness, and SportsArt ellipticals. Repeated-measures ANOVAs $(5 \times 1)$ identified forefoot and heel differences across conditions. Findings: Maximum forefoot forces and peak pressures were significantly lower than walking for each elliptical condition with one exception (Life Fitness peak pressure). However, sustained elliptical pedal contact time contributed to forefoot pressure-time integrals and dosages (i.e., cumulative pressure during one minute of activity) not varying significantly amongst elliptical and walking conditions. Heel maximum forces and peak pressures were significantly lower than walking during all elliptical conditions except SportsArt. Heel contact time on SportsArt
\end{abstract}

Published in Clinical Biomechanics 80 (2020) 105142.

doi:10.1016/j.clinbiomech.2020.105142

Copyright (c) 2020 Elsevier Ltd. Used by permission.

Submitted 25 November 2019; accepted 29 July 2020; published 1 August 2020. 
and Octane exceeded walking, and SportsArt heel contact time exceeded Life Fitness. Heel pressure-time integral was greater on SportsArt compared to walking, Life Fitness, and True. Sports Art heel dosage exceeded Life Fitness and True.

Interpretation: While elliptical training's sustained double limb support diminished maximal forces and peak pressures under the forefoot and heel compared to walking, each ellipticals' pressure-time integral and dosage were not significantly lower than walking. These findings point to the importance of carefully initiating elliptical training programs to minimize tissue injury, particularly if sensory neuropathy is present.

Keywords: Plantar pressure, Elliptical, Exercise, Older adult, Rehabilitation

\section{Introduction}

Elliptical trainers are widely used in home, fitness, and rehabilitation settings to address walking and cardiorespiratory training goals of older adults (Burnfield et al., 2011; Buster et al., 2013; Hornby et al., 2012; Huisinga et al., 2011; Jackson et al., 2010; Nelson et al., 2011). Similarities in lower extremity muscle demands and joint motions between elliptical training and walking (Burnfield et al., 2010) have served as impetus for clinicians to use ellipticals to promote intense repetitive practice of a gait-like activity post neurologic injury or illness (Burnfield et al., 2011; Burnfield et al., 2018; Buster et al., 2013; Cesar et al., 2020; Huisinga et al., 2011). The capacity to engage upper and lower extremity muscles provides unique opportunities for customizing cardiorespiratory challenge while training (Burnfield et al., 2019).

Two unique aspects of elliptical training compared to walking are that both feet remain in constant contact with the pedals throughout the movement cycle and a fixed stride length can be pre-selected on many devices. The added stability of sustained double limb support has proven beneficial for some individuals with balance deficits or weakness (Burnfield et al., 2011; Irons et al., 2015). Sustained double limb support would also appear beneficial for dissipating vertical ground reaction force peaks during early and late stance periods of gait that can contribute to potentially deleterious plantar pressures (Burnfield et al., 2004; Nilsson and Thorstensson, 1989). However, the sustained contact could also pose a risk for plantar tissue injury. Indeed, previous research focused on a single brand of fitness technology 
(i.e., Life Fitness) identified significantly longer contact time between the forefoot and support surface during elliptical training compared to treadmill walking despite no significant differences in peak pressures (Burnfield et al., 2007). Additionally, repetitive cycling at the same stride length might be expected to limit variability in foot-pedal contact patterns across strides, leading to an increased risk of sustained pressures in particular regions. Prolonged exposure to pressure has been postulated as a factor that can lead to tissue injury (Hsi et al., 2002; Sauseng et al., 1999; Stess et al., 1997), an event that is particularly concerning in older adults with diabetic sensory neuropathy who may be unable to sense potentially deleterious pressures or who may lack the circulation required to repair the damage (Frykberg et al., 1998; Kanade et al., 2006; Shah and Mueller, 2012). Additionally, older adults with age-related thinning of their heel pads (Jahss et al., 1992) may find extended periods of pressure under the heel uncomfortable. Alternatively, it is plausible that the predictable nature of the fixed stride length could enable participants to explore variability in other domains (e.g., cadence or Center of Mass sway) that might alter force and pressure distribution across the plantar aspect of the foot while elliptical training.

Elliptical trainers vary notably in their designs and these differences would be expected to alter the biomechanical demands placed on users' bodies, including plantar pressures. Within a subset of variable step length ellipticals that observationally promote movement patterns similar to walking (Burnfield et al., 2010), features such as minimum and maximum step length and step height differ (Burnfield et al., 2011). These variations, combined with electromechanical variations when overcoming flywheel resistance, likely contribute to the kinematic and muscle demand variations that have been documented as users trained on the four ellipticals (Burnfield et al., 2010). For example, average forward trunk lean was significantly greater when using the True $\left(9.9^{\circ}\right)$, Octane $\left(9.5^{\circ}\right)$ and Life Fitness $\left(9.4^{\circ}\right)$ compared to walking $\left(3.0^{\circ}\right.$ ) (Burnfield et al., 2010). In contrast, the more upright posture of the trunk while using the SportsArt $\left(6.0^{\circ}\right)$ did not differ significantly from walking (Burnfield et al., 2010). Given the trunk, head and neck account for $\sim 58 \%$ of body mass (Winter, 1990), it is reasonable to assume based on static biomechanical estimates that forces and pressures under the forefoot might be heightened and those under the 
heel might be lessened when using a device that shifts trunk alignment anterior relative to the ankle joint center. Yet previous research has not examined if plantar pressures vary across different ellipticals (Burnfield et al., 2007; Marks et al., 2014).

The aim of the current exploratory study was to compare forefoot and heel plantar pressure patterns generated while older adults exercised on four commercial elliptical trainers and walked overground at a self-selected comfortable pace. We hypothesized that maximum forces and peak pressures under the forefoot and heel would be lower during elliptical training compared to walking given the sustained double limb support throughout the elliptical movement cycle (Perry and Burnfield, 2010). Despite lower maximum forces and peak pressures during elliptical training, we hypothesized that cumulative pressure experienced under the forefoot and heel within a single movement cycle (pressure-time integral) and across a time-normalized period (i.e., dosage of pressure accumulated over a minute) would be similar to walking as the extended time spent in double limb support during elliptical training would offset reductions in pressure amplitude. We hypothesized that maximum force and peak pressure under the forefoot would be heightened when using ellipticals that promoted greater forward trunk lean (i.e., Life Fitness, True, and Octane) compared to using a device that promoted a more upright trunk posture (i.e., SportsArt). In contrast, we hypothesized that maximum force and peak pressure under the heel would be lessened when using ellipticals that promoted greater forward trunk lean compared to using a device that promoted a more upright trunk posture. Given the risk of pressure injuries in older adults arising from diabetic sensory neuropathies and structural foot changes (e.g., foot deformities and heel pad thinning) (Jahss et al., 1992; Myerson and Shereff, 1989), understanding how plantar pressure patterns vary across commercial elliptical trainers is essential for guiding clinical decision-making related to the safe prescription of elliptical trainers for functional and cardiovascular gains. 


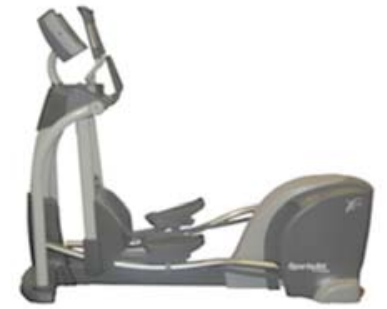

SportsArt

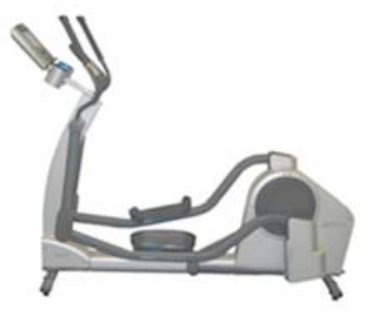

Life

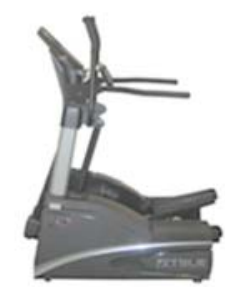

True

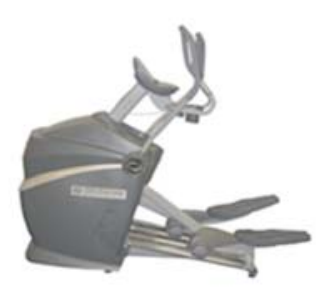

Octane

Fig. 1. Elliptical trainers studied.

\section{Methods}

\subsection{Participants}

Ten adults ( 6 men) between the ages of 60 and 80 years without reported neurologic, musculoskeletal or cardiovascular impairments that would affect their capacity to walk or exercise were recruited from the staff at Madonna Rehabilitation Hospitals (Lincoln, NE, United States) and the surrounding area. Their mean (SD, range) age, height, and mass were $68.1(4.5,61$ to 75$)$ years, $171.4(8.7,160$ to 185.4$) \mathrm{cm}$, and $76.1(21.6,54.5$ to 120.5$) \mathrm{kg}$, respectively.

\subsection{Instrumentation}

Four ellipticals with adjustable stride lengths were evaluated (Fig. 1). The SportsArt Fitness E870 (Woodinville, WA, United States; step length $=43$ to $74 \mathrm{~cm}$, horizontal separation between medial rims of pedals $=9 \mathrm{~cm}$, step height $=18 \mathrm{~cm}$ ) and Life Fitness X7 (Schiller Park, IL, United States; step length $=46$ to $61 \mathrm{~cm}$, horizontal separation between medial rims of pedals $=4 \mathrm{~cm}$, step height $=18 \mathrm{~cm}$ ) required participants to enter and exit from the side. In contrast, the Octane Fitness Pro450o (Brooklyn Park, MN, United States; step length $=46$ to $58 \mathrm{~cm}$, horizontal separation between medial rims of pedals $=6 \mathrm{~cm}$, step height $=19 \mathrm{~cm}$ ) and True Fitness Technology TSXa (St. Louis, MO, United States; step length $=43$ to $66 \mathrm{~cm}$, horizontal separation between medial rims of pedals $=5 \mathrm{~cm}$, step height $=18 \mathrm{~cm}$ ) allowed ingress and egress from behind each device. The four ellipticals included stationary and reciprocally moving handles. All participants 
elected to use the moveable handles which promoted reciprocal upper and lower extremity movement (i.e., when right pedal was most anterior then left handle was most posterior, and vice versa).

Walking trials were recorded as participants traversed a 10-m walkway. The middle six meters was designated for data collection to minimize the effects of acceleration and deceleration on participants' biomechanical variables. Data collection initiation and termination were triggered with E3G-MR19-US photoelectric sensors (Omron Inc., Schaumburg, Illinois, United States).

Plantar pressure data were recorded at a rate of $60 \mathrm{~Hz}$ using the Pedar system (Novel Electronics Inc., Munich, Germany) and the best fitting pair (length and width) selected from an inventory of 14 pairs of 2-mm-thick insoles. Each flexible insole contained 99 capacitive sensors to quantify pressure variables. Plantar pressure data were edited and evaluated using Emedlink and Multimask Evaluation software (Novel Electronics, Inc., Munich, Germany), respectively.

\subsection{Procedures}

All testing occurred in the Movement and Neurosciences Center located in the Institute for Rehabilitation Science and Engineering within Madonna Rehabilitation Hospitals. Before beginning the study, each participant signed a written informed consent approved by Madonna Rehabilitation Hospitals' Institutional Review Board. Participants were instructed to wear clothing and shoes they would normally use during exercise.

Each subject completed two familiarization sessions prior to the data collection session. During the first familiarization session, basic anthropometrics (age, height, weight) and lower limb dominance (foot used to kick a ball) were documented. Then, participants walked across the walkway at their self-selected comfortable speed. Participants performed walking trials until ten were matched to within 5\% of the average velocity (calculated from total time to traverse the sixmeter walkway). On average, 11 walking trials (range $=10$ to 13 ) were needed to achieve 10 trials of comparable speed.

Participants then elliptical trained on each device at a speed and stride length they perceived they would use during a typical workout. No effort was made to impose a consistent stride length across 
ellipticals or users. After a minimum of three minutes of elliptical training at the self-selected pace, participants rested up to five minutes and then were oriented to and repeated the procedure on a different elliptical. The order of training across ellipticals was randomized using a Matlab (MathWorks, Natick, MA, United States) program. Following use of the fourth elliptical, the first familiarization session ended and a second familiarization session was scheduled during which the walking and elliptical training activities were repeated.

Plantar pressure data collection was then performed on a separate session. Participants returned to the laboratory on a separate day (with the same pair of shoes used during familiarization) to complete the plantar pressure assessment. Prior to data collection, appropriately sized insoles (from a selection of 14 pairs) were placed in each participant's shoes between the insole and the participant's sock covered foot. Participants then laced their shoes to the desired tightness. Consistent with the manufacturer's guidelines, pressure insoles were calibrated by lifting each foot from the ground to establish a zero pressure baseline for the unloaded insole. Then, participants walked and elliptical trained using procedures similar to those for the familiarization sessions, except that Pedar data were recorded during the walking and elliptical trials, and participants exercised for only two minutes on each device once self-selected step length and cadence were achieved. Self-selected walking speed trials were purposefully performed first given these were not expected to fatigue older adults without known disability (Waters and Mulroy, 1999). Then, consistent with the familiarization sessions, the four elliptical trials were performed in an order randomized using Matlab. To reduce the potential impact of fatigue on elliptical performance, participants were allowed 5 min of rest between each trial, if needed.

\subsection{Data analysis}

Pedar data were initially screened and divided into steps for each walking trial and cycles for each elliptical trial using Emedlink software (Novel Electronics, Inc.). Walking cycles were defined from the first instant of stance phase pressure to the next onset of stance phase pressure for the reference limb. The characteristic elliptical cycle pressure pattern includes a period of high pressure during the downward 


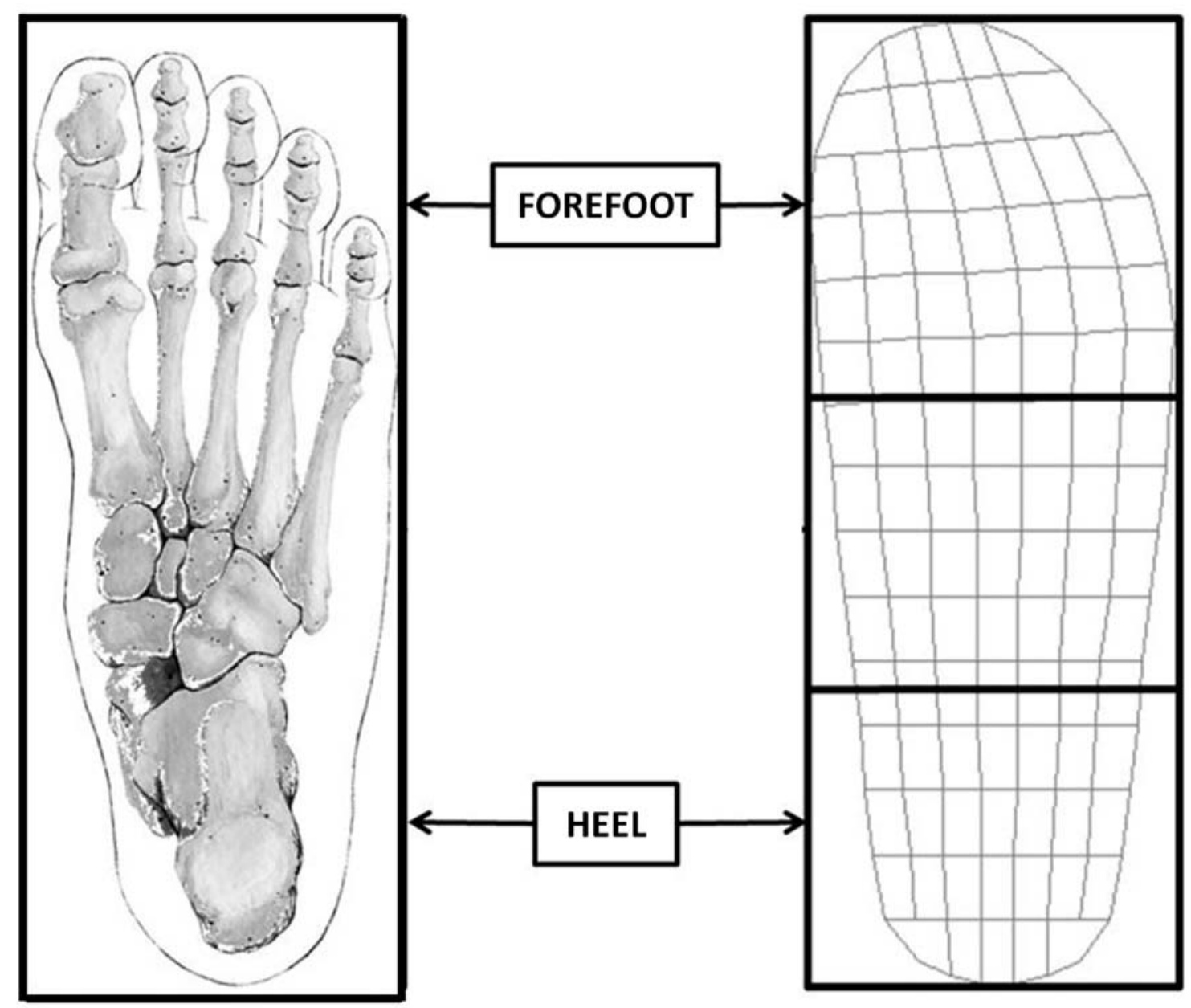

Fig. 2. Three anatomical foot regions divided with the manufacturer-provided masking algorithm. The two regions of interest for this study were the forefoot (distal $40 \%$ of longitudinal foot length) and heel (proximal 30\% of longitudinal foot length).

push phase and a pressure minimum during upward elevation of the reference pedal. Elliptical cycles were delineated as the period between successive pressure minima for the reference limb (Burnfield et al., 2007).

Novel Multitask Evaluation software (Novel Electronics, Inc.) was used to divide the foot mask into three anatomical areas (forefoot, arch, and heel) using a manufacturer-provided masking routine (Percent Mask Insole-3; Fig. 2) (Burnfield et al., 2007). The anatomical mask regions that served as the focus for this study were the forefoot 
(distal $40 \%$ of longitudinal foot length) and the heel (proximal 30\% of longitudinal foot length). Separate regional analyses of plantar pressure variables were then performed for the forefoot and heel regions. Analysis and hypothesis testing used the data recorded for the dominant limb during the final minute of each exercise. All participants demonstrated right lower-limb dominance.

For each participant, five pressure variables were calculated within the forefoot and the heel across the walking and four elliptical conditions. Mean maximum force identified the average of the maximum force (k) calculated across the cycles. Mean maximum peak pressure $(\mathrm{kPa})$ was the average of the peak pressures recorded during the series of movement cycles. Contact time reflected the time (expressed as $\%$ of cycle) that at least one sensor within the designated mask was activated during the movement cycle. Pressure-time integral $\left(\mathrm{kPa}^{*} \mathrm{~s}\right)$ identified the amount of pressure experienced during a stride or cycle. Pressure dosage reflected the cumulative pressure experienced in a given region per minute of walking or elliptical training $(\mathrm{kPa} / \mathrm{min})$ and was calculated by determining the number of gait/elliptical cycles that occurred in a minute and then multiplying by the pressure time integral. Pressure dosage extends interpretation of pressure time integral to factor in the impact of differing training speeds on the cumulative pressure a foot would experience during a minute of training on each device. For example, if two pressure time integrals were similar when using two ellipticals (e.g., $90 \mathrm{kPa} * \mathrm{~s}$ ), but a user trained twice as fast on one device compared to the other (e.g., 40 vs. 20 cycles per minute), then pressure dosage over one minute would be twice as great at the faster speed (i.e., 3600 vs. $1800 \mathrm{kPa} / \mathrm{min}$ ). Thus, pressure dosage normalizes for differing cycling rates and allows for comparison of the cumulative amount of pressure experienced by a foot region over a one-minute period regardless of self-selected cycling rate during walking or elliptical training.

\subsection{Statistical analysis}

Descriptive statistics were calculated for key variables using SigmaPlot 11.0 software (Systat, Chicago, IL, United States) and Excel ${ }^{\circledR}$ (Microsoft Corporation, Redmond, WA, United States). All data were initially screened for normality using the Shapiro-Wilk Test. If normality 
assumptions were accepted, then a separate one-way analysis of variance with repeated measures $(5 \times 1)$ was performed for each dependent measure (i.e., maximum force, peak pressure, contact time, pressure-time integral and pressure dosage) under the forefoot and again under the heel across the five conditions (Walking, Life Fitness, True, Octane and SportsArt). If the main effect was significant, then pairwise multiple comparisons were performed using Holm-Sidak method to determine which conditions differed from each other. If screening for a dependent variable revealed normality assumptions were violated, then data were transformed to ranks and the Friedman repeated measures ANOVA on ranks was used to identify a significant main effect across the five conditions. Pairwise multiple comparisons were then performed on the ranked data using the Tukey Test. To guide interpretation of the findings and sample size selection of future studies, a posteriori sample size calculations were performed for any dependent measure failing to demonstrate a statistically significant difference.

\section{Results}

\subsection{Training characteristics (Table 1)}

Participants' average self-selected comfortable walking speed of 72.9 $\mathrm{m} / \mathrm{min}$ arose from an average gait cycle frequency of 55 strides/ min and stride length of $1.37 \mathrm{~m}$. The average cycle rate (i.e., number of full revolutions per minute) during elliptical training ranged from 41 to 49 cycles/min, with the self-selected training stride length ranging from 0.97 to $1.01 \mathrm{~m}$.

Table 1 Spatiotemporal Characteristics during Walking and Training on Different Elliptical Devices, Mean (SD).

\begin{tabular}{lccc} 
Activity & Velocity (m/min) & Cycles/Minute & Stride Length $(\mathrm{m})$ \\
\hline Walking & $72.9(5.6)$ & $55(8)$ & $1.37(0.08)$ \\
Life Fitness & $\mathrm{n} / \mathrm{a}$ & $47(6)$ & $0.97(0.07)$ \\
True & $\mathrm{n} / \mathrm{a}$ & $49(6)$ & $0.98(0.10)$ \\
Octane & $\mathrm{n} / \mathrm{a}$ & $45(6)$ & $1.01(0.09)$ \\
SportsArt & $\mathrm{n} / \mathrm{a}$ & $41(4)$ & $0.99(0.17)$ \\
\hline
\end{tabular}




\subsection{Forefoot (Table 2; Fig. 3 upper graph)}

Maximum force under the forefoot was significantly lower during each elliptical condition compared to walking $(P<0.001$ for all pairwise comparisons); however, no significant differences in maximum force were recorded amongst ellipticals. Forefoot peak pressure was significantly lower during each elliptical condition except Life Fitness compared to walking ( $P<0.05$ for all pairwise comparisons); however, no significant differences in peak pressure were identified between ellipticals. Forefoot contact time ranged from 98 to 100\% of the elliptical cycle across the four devices compared to only $67 \%$ of the gait cycle; differences between each elliptical condition and walking were significant except True ( $P<0.05$ for all pairwise comparisons). Fig. 3 (upper graph) highlights the change in peak forefoot pressure across a single movement cycle for each elliptical and walking condition. As expected, forefoot plantar pressures registered zero during gait's swing period in contrast to the sustained pressures recorded during a similar period of the elliptical training movement cycle. With the number of participants available, no significant differences in forefoot pressure-time integral and pressure dosage could be detected across the five conditions. A post hoc sample size analysis indicated that ensuring adequate power to detect statistical significance in forefoot pressure-time integral and pressure dosage measures across the five conditions would require 33 and 16 participants, respectively.

Table 2 Forefoot variations in maximum force, peak pressure, contact time, pressure-time integral, and dosage across conditions, mean (SD) [Median].

\begin{tabular}{|c|c|c|c|c|c|}
\hline Activity & $\begin{array}{l}\text { Maximum } \\
\text { Force }(N)\end{array}$ & $\begin{array}{c}\text { Peak Pressure } \\
(\mathrm{kPa})\end{array}$ & $\begin{array}{c}\text { Contact Time } \\
(\% \text { Cycle })\end{array}$ & $\begin{array}{l}\text { Pressure-Time } \\
\text { Integral }\left(\mathrm{kPa}^{*} \mathrm{~s}\right)\end{array}$ & $\begin{array}{c}\text { Dosage } \\
(\mathrm{kPa} / \mathrm{min})\end{array}$ \\
\hline Walking & $802(223)[729]$ & $223(38)[214]$ & $67(13)[64]$ & $72(16)[66]$ & 3699 (962) [3458] \\
\hline Life Fitness & $541(153)[564]$ & $150(39)[163]$ & $100(0)[100]$ & 89 (23) [84] & 4376 (1347) [4321] \\
\hline True & $477(184)[478]$ & $153(88)[136]$ & $98(5)[100]$ & $90(44)[78]$ & $4553(2182)[4278]$ \\
\hline Octane & $461(150)[475]$ & $126(43)[130]$ & $100(0)[100]$ & $83(24)[86]$ & $3710(1120)$ [4064] \\
\hline SportsArt & $460(171)[435]$ & $132(52)[128]$ & $99(4)[100]$ & $84(25)[82]$ & 3516 (1096) [3423] \\
\hline Main Effect across & $\mathrm{W}>\mathrm{S}, \mathrm{O}, \mathrm{T}, \mathrm{L}$ & $\mathrm{W}>\mathrm{O}, \mathrm{S}, \mathrm{T}$ & $\mathrm{L}, \mathrm{O}, \mathrm{S}>\mathrm{W}$ & NS a & NS b \\
\hline All Conditions $(5 \times 1)$ & $P<0.001, \mathrm{~F}=19.92$ & $P<0.001, \chi^{2}=22.64$ & $P<0.001, \chi^{2}=23.82$ & $P=0.62, \chi^{2}=2.64$ & $P=0.16, \chi^{2}=6.64$ \\
\hline
\end{tabular}

Abbreviations: W, overground walking; L, Life Fitness X7; T, True Fitness Technology TSXa; O, Octane Fitness Pro450o; S, SportsArt Fitness E870; NS, not significant.

a. Post-hoc sample size analysis suggested 33 individuals were required to provide adequate power to detect statistical differences.

b. Post-hoc sample size analysis suggested 16 individuals were required to provide adequate power to detect statistical differences. Note: $\mathrm{F}$ values indicate use of ANOVA with repeated measures given normally distributed data, and $\chi^{2}$ values indicate use of Friedman repeated measures ANOVA on ranks given non-normally distributed data. 

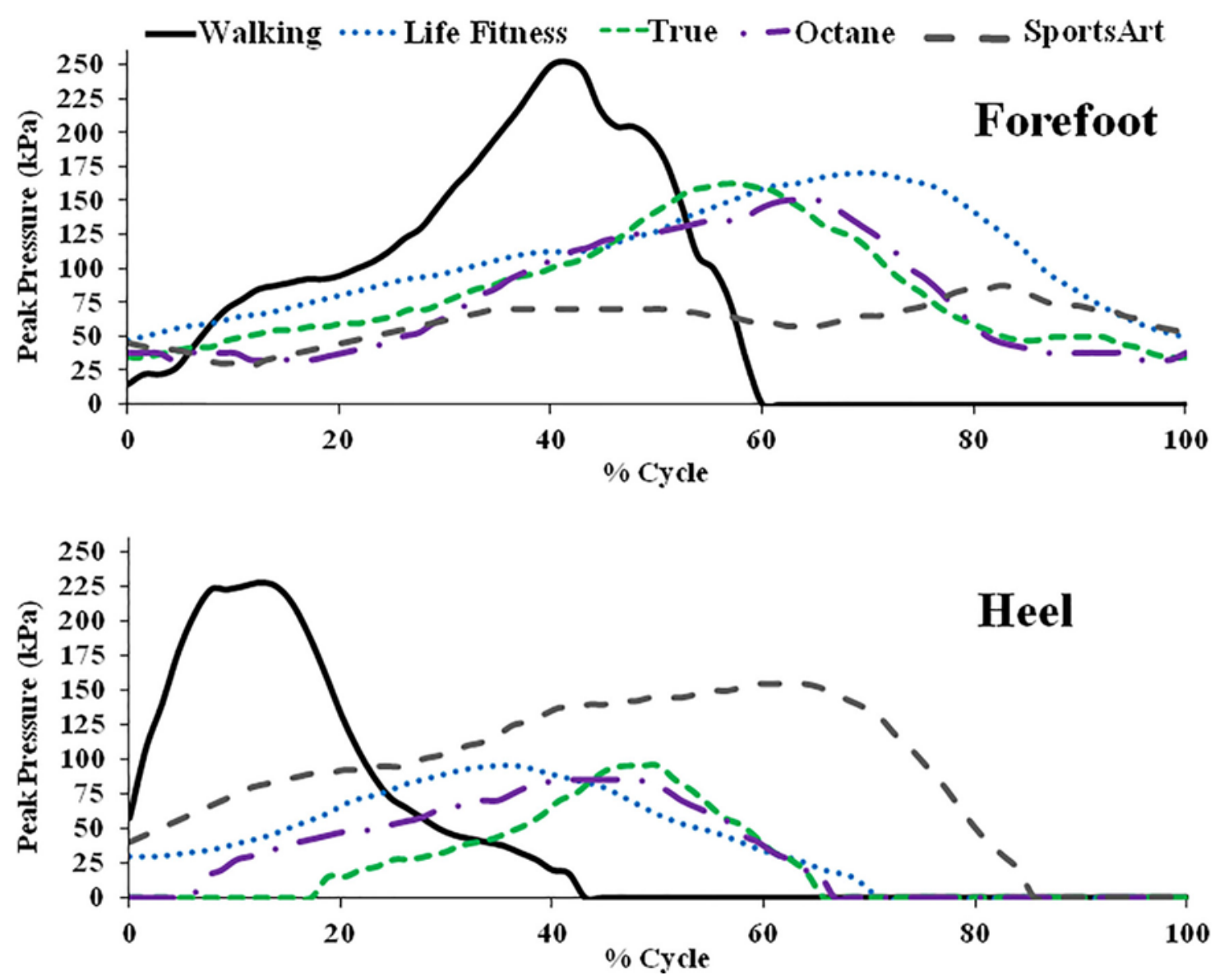

Fig. 3. Exemplar plantar pressure time series during walking and exercising on the four ellipticals.

\subsection{Heel (Table 3; Fig. 3 lower graph)}

Maximum force and peak pressure under the heel were significantly lower during each elliptical condition except SportsArt compared to walking $(P<0.05$ for all pairwise comparisons); however, no significant differences in these variables were detected amongst ellipticals. Contact time was longer when exercising on SportsArt and Octane compared to walking; and also longer when using SportsArt compared to Life Fitness ( $P<0.001$ for all pairwise comparisons). Fig. 3 (lower) highlights the change in average heel peak pressure across time for each elliptical and walking condition. In contrast to the abrupt rise in plantar pressure under the heel as weight rapidly loaded onto the limb during gait, elliptical training was characterized by a more gradual 
Table 3 Heel Variations in Maximum Force, Peak Pressure, Contact Time, Pressure-Time Integral, and Dosage Across Conditions, Mean (SD) [Median].

\begin{tabular}{|c|c|c|c|c|c|}
\hline Activity & $\begin{array}{l}\text { Maximum } \\
\text { Force }(N)\end{array}$ & $\begin{array}{c}\text { Peak Pressure } \\
\text { (kPa) }\end{array}$ & $\begin{array}{c}\text { Contact Time } \\
(\% \text { Cycle })\end{array}$ & $\begin{array}{l}\text { Pressure-Time } \\
\text { Integral }\left(\mathrm{kPa}^{*} \mathrm{~s}\right)\end{array}$ & $\begin{array}{c}\text { Dosage } \\
(\mathrm{kPa} / \mathrm{min})\end{array}$ \\
\hline Walking & $576(138)[556]$ & $188(35)[179]$ & $58(18)[59]$ & 49 (11) $[48]$ & $2471(450)$ [2454] \\
\hline Life Fitness & o) [339] & $122(34)[124]$ & $68(2$ & $48(21)[45]$ & $2304(1089)$ [1836] \\
\hline True & $355(1$ & $126(54)[116]$ & 3) $[66]$ & $51(30)[37]$ & 2645 (1685) [1803] \\
\hline Octane & $344(121)[311]$ & $116(29)[109]$ & 88 (18) [99] & $59(24)[49]$ & $2643(1142)$ [2371] \\
\hline SportsArt & 438 (125) [409] & $158(24)[155]$ & $96(5)[98]$ & $93(33)[87]$ & $4023(1852)[3530]$ \\
\hline Main Effect across & $\mathrm{W}>\mathrm{O}, \mathrm{T}, \mathrm{L}$ & $\mathrm{W}>\mathrm{O}, \mathrm{L}, \mathrm{T}$ & $\mathrm{S}, \mathrm{O}>\mathrm{W} \mathrm{S}>\mathrm{L}$ & $\mathrm{S}>\mathrm{L}, \mathrm{T}, \mathrm{W}$ & $\mathrm{S}>\mathrm{L}, \mathrm{T}$ \\
\hline All Conditions $(5 \times 1)$ & $P<0.001, \chi^{2}=27.84$ & $P<0.001, \chi^{2}=22.25$ & $P<0.001, \mathrm{~F}=7.55$ & $P<0.001, \chi^{2}=18.64$ & $P=0.02, \chi^{2}=12.16$ \\
\hline
\end{tabular}

Abbreviations: W, overground walking; L, Life Fitness X7; T, True Fitness Technology TSXa; O, Octane Fitness Pro4500; S, SportsArt Fitness E870.

F values indicate use of ANOVA with repeated measures given normally distributed data, and $\chi^{2}$ values indicate use of Friedman repeated measures ANOVA on ranks given non-normally distributed data.

rise in heel pressure. Pressure-time integral under the heel was significantly lower when using Life Fitness and True and during walking compared to Sports-Art ( $P<0.05$ for all pairwise comparisons). Pressure dosage under the heel was significantly lower on Life Fitness and True compared to SportsArt ( $P<0.05$ for all pairwise comparisons).

\section{Discussion}

The aging "baby boomer" population and growing emphasis on maintaining a physically active lifestyle are contributing to an expanded number of older adults using cardiovascular exercise equipment such as ellipticals in fitness and home settings. Beyond that, the similarity to gait of movement patterns while exercising on some ellipticals has resulted in growing use within rehabilitation to promote task-related training following neurologic injury or illness (Burnfield et al., 2011; Burnfield et al., 2018; Buster et al., 2013; Cesar et al., 2020; Huisinga et al., 2011). Findings from this study enhance understanding of how therapeutic exercise device selection may be used to promote safer and more comfortable exercise experiences for older adults, including the approximately one-quarter of Americans age 65 and older who may lack protective sensation due to diabetes (Centers for Disease Control and Prevention, 2017).

Consistent with our first hypothesis, the sustained double limb support of elliptical training lowered maximum forces and peak pressures 
during each elliptical condition compared to walking for both the forefoot (maximum forces 33 to $43 \%$ lower, peak pressures 31 to $43 \%$ lower) and heel (maximum forces 24 to $40 \%$ lower, peak pressures 16 to $38 \%$ lower), although differences did not always achieve statistical significance (i.e., Life Fitness forefoot peak pressure; SportsArt heel maximum force and peak pressure). While both activities allow for a trailing limb posture, the sustained double limb support of elliptical training diminishes the typical second peak in ground reaction force that arises during gait's terminal stance as the plantar flexors contract vigorously to control body weight's progression over the forefoot (Burnfield et al., 2010). The sustained double limb support of elliptical training also diminishes the first ground reaction force peak that typically occurs under the heel during abrupt loading of bodyweight onto the outstretch limb during weight acceptance.

The current study's finding of significant reductions in peak pressure under the forefoot while using the Octane, SportsArt and True has important clinical implications, particularly in the older population. With the prevalence of diabetes projected to grow during the upcoming decade, there will be a greater number of older adults living with the long term consequences of diabetes including peripheral neuropathies. Loss of protective sensation places individuals with sensory neuropathy at greater risk for developing diabetic pressure ulcers/injuries particularly when the foot is exposed repetitively to elevated pressures (Frykberg et al., 1998). While an evidenced-based threshold for safe pressures has yet to be established, a clinicallyderived threshold of maintaining peak pressures $\leq 210 \mathrm{kPa}$ is often cited to reduce risk of pressure injuries in individuals lacking protective sensation (Mueller et al., 1999). In the current study, the average peak pressure experienced under the forefoot while training on each elliptical was well below the $\leq 210 \mathrm{kPa}$ threshold. In contrast, the average forefoot peak pressure during walking exceeded the threshold suggesting that some ellipticals may provide a safer alternative to walking in situations where mitigating high pressures is critical. A prospective study of individuals with diabetes, identified the risk of foot ulcer recurrence was significantly reduced when peak pressures within prescription footwear were maintained below $200 \mathrm{kPa}$ and footwear was worn for greater than $80 \%$ of steps taken (Waaijman et al., 2014), pointing to the importance of having at-risk individuals 
wear appropriately fit prescription footwear and orthotics. Beyond sensory loss, some older adults and individuals with diabetes may also experience changes to their foot structure (e.g., claw/hammer toes (Myerson and Shereff, 1989) or thinning of plantar fat pads (Jahss et al., 1992)). While not elevated compared to walking, the finding that forefoot peak pressures while using the Life Fitness elliptical were not significantly lower than walking also points to the need to educate users who may lack protective sensation regarding the importance of checking their skin frequently when first starting to use the Life Fitness elliptical or when walking.

Despite generally lower maximum forces and peak pressures during elliptical training compared to walking, the forefoot's and heel's pressure- time integral and pressure dosage during elliptical training did not differ significantly or actually exceeded those arising during walking (i.e., heel pressure-time integral using SportsArt exceeded walking). Collectively, these findings indicate that the extended time spent during elliptical training in double limb support (forefoot contact time ranged from 98 to $100 \%$ cycle across ellipticals vs. $67 \%$ during gait) offset reductions in pressure amplitude. This finding has important clinical implications. Sustained pressure (even low amplitude) over at-risk tissue can lead to development of pressure injuries (Gefen, 2009a; Gefen, 2009b). Thus, in the presence of conditions where reducing not only the peak pressures, but also the cumulative load experienced by the foot is critical, close monitoring of the foot's response to exercise (whether walking or elliptical training) is critical. This could include checking the skin on the plantar aspect for signs of redness, blistering, or abrasion prior to, during, and following early training sessions and stopping training if areas of concern are identified. For those with flexibility or vision challenges, integration of a caregiver/clinician or use of a magnifying mirror may facilitate skin inspections. Wearing white socks could also facilitate identification of skin abrasions given observable evidence of drainage/blood.

The final two hypotheses exploring if maximum forces and peak pressures were greater under the forefoot and, vice versa less under the heel, when using devices that promoted a greater forward trunk lean (i.e., True, Octane, and Life Fitness)(Burnfield et al., 2010) were not supported. A sub-analysis of kinematic data for the 10 participants in the current study was extracted from a previously reported 
larger data set (Burnfield et al., 2010). Similar to the larger data set of 20 participants, forward trunk lean on average was greater when the 10 older adults used the True $\left(13.6^{\circ}\right)$, Octane $\left(13.5^{\circ}\right)$ and Life Fitness $\left(12.7^{\circ}\right)$ compared to SportsArt $\left(9.7^{\circ}\right)$. In the current study, it is conceivable that greater forward trunk leans while using the True, Octane and Life Fitness devices were compensated for by shifting the Centre of Mass backward, thus altering the expected impact of the forward trunk lean on forefoot and heel maximum forces and peak plantar pressures across ellipticals. Future research, exploring the impact of pre-specified postural alignments (e.g. $10^{\circ}$ forward vs. $10^{\circ}$ backward trunk lean) on Centre of Mass location and plantar loading patterns could help elucidate if postural alignment can be used to consistently and meaningfully diminish plantar pressures under load-sensitive areas of the foot during elliptical training. In addition, it is likely that subtle differences in device design (e.g., weight, location and inertial properties of the flywheel, inter-pedal spacing distance in the frontal plane, mechanical resistance within each device's linkages, and pedal height excursion variations) also contribute to differences in plantar pressure patterns. With the advent of a motor-assisted elliptical (Burnfield et al., 2019; Irons et al., 2015; Nelson et al., 2011), future research could also explore the impact of motor-assistance on plantar pressures as training at a specified speed using the motor's assistance would be expected to control for mechanical resistance within the device's linkages.

Our findings must be interpreted with care as a limitation to this work is related to the sample size. Although 8 out of 10 comparisons across conditions (i.e., ellipticals and walking) reached statistical significance, an a posteriori sample size analysis (alpha level set at 0.05 and a minimum of $80 \%$ power) determined that 33 participants were required to provide adequate power to detect differences for forefoot pressure-time integral and 16 participants for forefoot dosage. Another study limitation was the use of handles during the elliptical conditions as the mechanical linkage between foot pedals and upperextremity handles creates a proscriptive reciprocal movement pattern that allows force to transfer between limbs and potentially alter plantar pressure patterns. Future research could help elucidate the impact of different elliptical training hand positions (e.g., stationary, reciprocally moving, no handles) on lower extremity plantar pressure 
patterns. Finally, the impact of elliptical training at a consistent/fixed stride length on plantar pressure variability across strides is still unknown. Previous research identified greater variability in ankle and knee kinematics, but lesser at the hip while elliptical training on the True elliptical compared to treadmill walking in younger adults (with and without traumatic brain injury); however, no analyses were performed across other ellipticals (Buster et al., 2013). If movement variability is greater during elliptical training on some devices compared to others, then it is conceivable that the specific location of maximum force and peak pressure within a foot mask might shift across strides (e.g., from first to third to fifth metatarsal head in forefoot mask) thus deconcentrating potentially deleterious pressures across strides. Future research could help guide understanding of the variability of plantar pressure patterns across ellipticals and stride lengths in older adults with and without neuropathy.

Even though our work was conducted with individuals without known chronic conditions or disability, the knowledge obtained has implications for individuals with medical conditions associated with sensory neuropathies or peripheral vascular diseases. Engaging older adults in safe aerobic exercise is important not only for improving bone and muscle strength, but also for helping control body weight, another factor critical for long-term function and health (Piercy et al., 2018) and a common co-morbidity in diabetes. Position statements by the American College of Sports Medicine (Riebe et al., 2018) and other influential organizations such as the American Diabetes Association (Colberg et al., 2016) outline key guidelines that include recommendations for individuals to engage in exercise up to 7 days per week to achieve targeted cardiorespiratory, body composition, and blood glucose goals. Thus, providing options for older adults to exercise safely to increase musculoskeletal and cardiovascular health is of great clinical importance. As higher plantar pressures increase the risk for diabetic foot ulceration (Frykberg et al., 1998; Stess et al., 1997) and amputation (Borg et al., 2018; Bus et al., 2009; Fernando et al., 2014), results from our work can guide clinical decision-making regarding how to incorporate available elliptical trainers into therapeutic/aerobic exercise programs. 


\section{Conclusion}

While elliptical training's sustained double limb support diminished maximal forces and peak pressures under the forefoot and heel compared to walking, the cumulative pressure dosage during each elliptical condition was not significantly lower than walking. These findings point to the importance of carefully initiating elliptical training programs to minimize tissue injury, particularly if sensory neuropathy is present.

$$
\ddagger \neq \ddagger
$$

Funding The contents of this research were developed, in part, under a Grant from the United States Department of Education, National Institute on Disability and Rehabilitation Research (H133070209; Principal Investigator: Burnfield). However, the contents do not necessarily represent the policy of the Department of Education, and endorsement by the Federal Government should not be assumed.

Author contributions Judith M. Burnfield and Thad W. Buster made substantial contributions to the funding acquisition, conception, design of the study, and project administration. All three authors made substantial contributions to the analysis and interpretation of data, drafting and revising the manuscript critically for important intellectual content, and provided final approval of the version to be submitted.

Declaration of Competing Interest Three patents (2 US and 1 Canadian) have been issued to J. M. Burnfield and T. W. Buster for a motor-assisted elliptical that was developed in association with the above referenced grant. The patented technology has been licensed to SportsArt for commercial distribution and any sales could lead to a royalty distribution. SportsArt manufactured one of the ellipticals discussed in this research study. Guilherme M. Cesar: None.

\section{References}

Borg, J., Mizzi, S., Formosa, C., 2018. Peak pressure data and pressuretime integral in the contralateral limb in patients with diabetes and a trans-tibial prosthesis. Gait Posture 64, 55-58. https://doi.org/10.1016/j. gaitpost.2018.05.023

Burnfield, J.M., Few, C.D., Mohamed, O.S., Perry, J., 2004. The influence of walking speed and footwear on plantar pressures in older adults. Clin. Biomech. 19, 78-84. https://doi.org/10.1016/j.clinbiomech.2003.09.007 
Burnfield, J.M., Jorde, A.G., Augustin, T.R., Augustin, T.A., Bashford, G.R., 2007. Variations in plantar pressure variables across five cardiovascular exercises. Med. Sci. Sports Exerc. 39, 2012-2020. https://doi.org/10.1249/ mss.obo13e318148bdfa

Burnfield, J.M., Shu, Y., Buster, T., Taylor, A., 2010. Similarity of joint kinematics and muscle demands between elliptical training and walking: implications for practice. Phys. Ther. 90, 289-305. https://doi.org/10.2522/ptj.20090033

Burnfield, J.M., Shu, Y., Buster, T.W., Taylor, A.P., Nelson, C.A., 2011. Impact of elliptical trainer ergonomic modifications on perceptions of safety, comfort, workout, and usability for people with physical disabilities and chronic conditions. Phys. Ther. 91, 1604-1617. https://doi.org/10.2522/ptj.20100332

Burnfield, J.M., Cesar, G.M., Buster, T.W., Irons, S.L., Pfeifer, C.M., 2018. Walking and fitness improvements in child with diplegic cerebral palsy following motor-assisted elliptical intervention. Ped Phys Ther 30, E1-E7. https://doi. org/10.1097/PEP.0000000000000541

Burnfield, J., Pfeifer, C., Kwapiszeski, S., Irons, S., Buster, T., Cesar, G., 2019. Impact of ICARE training speed and motor assistance on cardiovascular response. Cardiopul Phys Ther J 30, 115-122. https://doi.org/10.1097/ CPT.0000000000000098

Bus, S.A., van Deursen, R.W., Kanade, R.V., et al., 2009. Plantar pressure relief in the diabetic foot using forefoot offloading shoes. Gait Posture 29, 618-622. https://doi.org/10.1016/j.gaitpost.2009.01.003

Buster, T.W., Burnfield, J.M., Taylor, A.P., Stergiou, N., 2013. Lower extremity kinematics during walking and elliptical training in individuals with and without traumatic brain injury. J. Neurol. Phys. Ther. 37, 176-186. https://doi. org/10.1097/NPT.0000000000000022

Centers for Disease Control and Prevention, 2017. National Diabetes Statistics Report. Centers for Disease Control and Prevention - US Department of Health and Human Services, Atlanta, GA, pp. 2017.

Cesar, G.M., Buster, T.W., Burnfield, J.M., 2020. Cardiorespiratory fitness, balance and walking improvements in an adolescent with cerebral palsy (GMFCS II) and autism after motor-assisted elliptical training. Eur. J. Phys. 22, 124-132. https://doi.org/10.1080/21679169.2018.1536764

Colberg, S.R., Sigal, R.J., Yardley, J.E., et al., 2016. Physical activity/exercise and diabetes: a position statement of the American Diabetes Association. Diabetes Care 39, 2065-2079. https://doi.org/10.2337/dc16-1728

Fernando, M.E., Crowther, R.G., Pappas, E., et al., 2014. Plantar pressure in diabetic peripheral neuropathy patients with active foot ulceration, previous ulceration and no history of ulceration: a meta-analysis of observational studies. PLoS One 9, e99050. https://doi.org/10.1371/journal.pone.0099050

Frykberg, R.G., Lavery, L.A., Pham, H., Harvey, C., Harkless, L., Veves, A., 1998. Role of neuropathy and high foot pressures in diabetic foot ulceration. Diabetes Care 21, 1714-1719. https://doi.org/10.2337/diacare.21.10.1714 
Gefen, A., 2009a. Reswick and Rogers pressure-time curve for pressure ulcer risk. Part 1. Nurs. Stand. 23:64, 66, 68 passim. https://doi.org/10.7748/ ns2009.07.23.45.64.c7115

Gefen, A., 2009b. Reswick and Rogers pressure-time curve for pressure ulcer risk. Part 2. Nurs. Stand. 23, 40-44. https://doi.org/10.7748/ns2009.07.23.46.40. c7169

Hornby, T.G., Kinnaird, C.R., Holleran, C.L., Rafferty, M.R., Rodriguez, K.S., Cain, J.B., 2012. Kinematic, muscular, and metabolic responses during exoskeletal-, elliptical-, or therapist-assisted stepping in people with incomplete spinal cord injury. Phys. Ther. 92, 1278-1281. https://doi.org/10.2522/ptj.20110310

Hsi, W.L., Chai, H.M., Lai, J.S., 2002. Comparison of pressure and time parameters in evaluating diabetic footwear. Am J Phys Med Rehabil 81, 822-829. https:// doi.org/10.1097/00002060-200211000-00004

Huisinga, J.M., Filipi, M.L., Stergiou, N., 2011. Elliptical exercise improves fatigue ratings and quality of life in patients with multiple sclerosis. J. Rehabil. Res. Dev. 48, 881-89o. https://doi.org/10.1682/jrrd.2010.08.0152

Irons, S.L., Brusola, G.A., Buster, T.W., Burnfield, J.M., 2015. Novel motor-assisted elliptical training intervention improves six-minute walk test and oxygen cost for an individual with progressive supranuclear palsy. Cardiopulm Phys Ther J 26, 36-41. https://doi.org/10.1097/CPT.0000000000000027

Jackson, K., Merriman, H., Campbell, J., 2010. Use of an elliptical machine for improving functional walking capacity in individuals with chronic stroke: a case series. J. Neurol. Phys. Ther. 34, 168-174. https://doi.org/10.1097/ NPT.obo13e3181ee682C

Jahss, M.H., Michelson, J.D., Desai, P., et al., 1992. Investigations into the fat pads of the sole of the foot: anatomy and histology. Foot Ankle 13, 233-242. https:// doi.org/10.1177/107110079201300502

Kanade, R.V., van Deursen, R.W.M., Harding, K., Price, P., 2006. Walking performance in people with diabetic neuropathy: benefits and threats. Diabetologia 49, 1747-1754. https://doi.org/10.1007/s00125-006-0309-1

Marks, R., Beran, B., Long, J.T., et al., 2014. Plantar pressure analysis during rehabilitative exercise. Crit Rev Phys Rehabil Med 26, 193-201. https://doi. org/10.1615/CritRevPhysRehabilMed.2014011103

Mueller, M.J., Smith, K.E., Commean, P.K., Robertson, D.D., Johnson, J.E., 1999. Use of computed tomography and plantar pressure measurement for management of neuropathic ulcers in patients with diabetes. Phys. Ther. 79, 296-307 (PMID: 10078773).

Myerson, M.S., Shereff, M.J., 1989. The pathological anatomy of claw and hammer toes. J. Bone Joint Surg. Am. 71, 45-49 (PMID: 2913002).

Nelson, C.A., Burnfield, J.M., Shu, Y., Buster, T.W., Taylor, A., Graham, A., 2011. Modified elliptical machine motor-drive design for assistive gait rehabilitation. J Med Device 5 https://doi.org/10.1115/1.4003693.021001.1-7

Nilsson, J., Thorstensson, A., 1989. Ground reaction forces at different speeds of human walking and running. Acta Physiol. Scand. 136, 217-227. https://doi. org/10.1111/j.1748-1716.1989.tbo8655.X 
Perry, J., Burnfield, J.M., 2010. Gait Analysis: Normal and Pathological Function. Slack Incorporated, Thorofare, New Jersey.

Piercy, K.L., Troiano, R.P., Ballard, R.M., et al., 2018. The Physical Activity Guidelines for Americans, 2nd edition. 320. JAMA, pp. 2020-2028. https://doi. org/10.1001/jama.2018.14854

Riebe, D., Ehrman, J.K., Liguori, G., Magal, M., 2018. ACSM’s Guidelines for Exercise Testing and Prescription, 10th edition. Wolters Kluwer, Philadelphia, PA.

Sauseng, S., Kastenbauer, T., Sokol, G., Irsigler, K., 1999. Estimation of risk for plantar foot ulceration in diabetic patients with neuropathy. Diabetes Nutr Metab 12, 189-193. https://doi.org/10.1016/j.foot.2012.05.001

Shah, K.M., Mueller, M.J., 2012. Effect of selected exercises on in-shoe plantar pressures in people with diabetes and peripheral neuropathy. Foot 22, 130-134. https://doi.org/10.1016/j.foot.2012.05.001

Stess, R.M., Jensen, S.R., Mirmiran, R., 1997. The role of dynamic plantar pressures in diabetic foot ulcers. Diabetes Care 20, 855-858. https://doi. org/10.2337/diacare.20.5.855

Waaijman, R., de Haart, M., Arts, M.L., et al., 2014. Risk factors for plantar foot ulcer recurrence in neuropathic diabetic patients. Diabetes Care 37, 1697-1705. https://doi.org/10.2337/dc13-2470

Waters, R.L., Mulroy, S.J., 1999. The energy expenditure of normal and pathologic gait. Gait Posture 9, 207-231. https://doi.org/10.1016/sog66-6362(99)oooog-0

Winter, D.A., 1990. Biomechanics and Motor Control of Human Movement, 2nd ed. John Wiley \& Sons, Inc., Toronto. 